\title{
EULAR recommendations for terminology and research in individuals at risk of rheumatoid arthritis: report from the Study Group for Risk Factors for Rheumatoid Arthritis
}

\author{
Danielle M Gerlag, ${ }^{1}$ Karim Raza, ${ }^{2}$ Lisa G M van Baarsen, ${ }^{1}$ Elisabeth Brouwer, ${ }^{3}$ \\ Christopher D Buckley, ${ }^{2}$ Gerd R Burmester, ${ }^{4}$ Cem Gabay, ${ }^{5}$ Anca I Catrina, ${ }^{6}$ \\ Andrew P Cope, ${ }^{7}$ François Cornelis, ${ }^{8}$ Solbritt Rantapää Dahlqvist, ${ }^{9}$ Paul Emery, ${ }^{10}$ \\ Stephen Eyre, ${ }^{11}$ Axel Finckh, ${ }^{5}$ Steffen Gay, ${ }^{12}$ Johanna M Hazes, ${ }^{13}$ \\ Annette van der Helm-van Mil, ${ }^{14}$ Tom W J Huizinga, ${ }^{14}$ Lars Klareskog, ${ }^{6}$ Tore K Kvien, ${ }^{15}$ \\ Cathryn Lewis, ${ }^{7}$ Klaus P Machold, ${ }^{16}$ Johan Rönnelid, ${ }^{17}$ Dirkjan van Schaardenburg, ${ }^{18}$ \\ Georg Schett, ${ }^{19}$ Josef S Smolen, ${ }^{16}$ Sue Thomas, ${ }^{20}$ Jane Worthington, ${ }^{11}$ Paul P Tak ${ }^{1}$
}

For numbered affiliations see end of article

\section{Correspondence to Danielle M Gerlag, Division of Clinical Immunology and Rheumatology, Academic Medical Center/University of Amsterdam, F4-105 PO Box 22700, $1100 \mathrm{DE}$ Amsterdam, The Netherlands; d.m.gerlag@amc.uva.nl}

\section{Handling editor}

Johannes WJ Bijlsma

Received 1 November 2011 Accepted 23 January 2012 Published Online First 2 March 2012

\section{ABSTRACT}

The Study Group for Risk Factors for Rheumatoid Arthritis was established by the EULAR Standing Committee on Investigative Rheumatology to facilitate research into the preclinical and earliest clinically apparent phases of rheumatoid arthritis (RA). This report describes the recommendation for terminology to be used to define specific subgroups during different phases of disease, and defines the priorities for research in this area. Terminology was discussed by way of a three-stage structured process: A provisional list of descriptors for each of the possible phases preceding the diagnosis of RA were circulated to members of the study group for review and feedback. Anonymised comments from the members on this list were fed back to participants before a 2-day meeting. 18 participants met to discuss these data, agree terminologies and prioritise important research questions. The study group recommended that, in prospective studies, individuals without RA are described as having: genetic risk factors for RA; environmental risk factors for RA; systemic autoimmunity associated with RA; symptoms without clinical arthritis; unclassified arthritis; which may be used in a combinatorial manner. It was recommended that the prefix 'pre-RA with:' could be used before any/any combination of the five points above but only to describe retrospectively a phase that an individual had progressed through once it was known that they have developed RA. An approach to dating disease onset was recommended. In addition, important areas for research were proposed, including research of other tissues in which an adaptive immune response may be initiated, and the identification of additional risk factors and biomarkers for the development of RA, its progression and the development of extraarticular features. These recommendations provide guidance on approaches to describe phases before the development of RA that will facilitate communication between researchers and comparisons between studies. A number of research questions have been defined, requiring new cohorts to be established and new techniques to be developed to image and collect material from different sites.
Rheumatoid arthritis (RA) is a prototype immunemediated inflammatory disease, characterised by a symmetric polyarthritis usually involving the small joints of the hands and feet. Other joints can also be involved. Rheumatoid factor (RF) and/or anticitrullinated protein antibody (ACPA) positive ('seropositive') RA is associated with more aggressive articular disease, a higher frequency of extraarticular manifestations and increased mortality. ${ }^{1}$ RA is still associated with significant morbidity despite major developments in antirheumatic therapy. Among the inflammatory joint diseases, RA is the commonest and the most important in socioeconomic terms.

Over the past few years, research in the field of RA has focused on the earliest stages of disease, leading to the discovery that circulating autoantibodies, specifically immunoglobulin M-RF and ACPA, and increased acute phase reactants precede the clinical onset of the disease. ${ }^{2-5}$ These autoantibodies are present a median of 5 years before clinical symptoms appear. ${ }^{3}$ Subjects with arthralgia and these autoantibodies have an approximately 30\% chance of developing RA within 1 year. ${ }^{6}$ These data form strong evidence that clinical signs and symptoms may be preceded by a preclinical phase for several years, and that the preclinical and earliest clinically apparent phases of RA are likely to represent important therapeutic windows within which clinical outcomes can be dramatically modulated.7 Factors such as smoking, which act during the preclinical phase, leading to citrullination of peptides and ACPA formation, ${ }^{8}$ are increasingly being recognised.

To facilitate research in this area, the European League Against Rheumatism (EULAR) Standing Committee on Investigative Rheumatology established a study group in February 2011 to build a European network of excellence. Key aims of this study group were to develop a more accurate understanding of the mechanisms driving the immunological abnormalities seen during the preclinical phase of RA and the stimuli that turn such abnormalities into a joint-centric disease, in online under the BMJ Journals unlocked scheme, see http:// ard.bmj.com/info/unlocked.dtl 
Box 1. Information provided to delegates for anonymous comment: summaries of phases that individuals (may) pass through before the development of RA. Delegates were asked to provide descriptive terms for these phases and to comment on their chosen descriptive terms.

- Individual at risk of RA on the basis of genetic and environmental risk factors with no identifiable laboratory abnormalities and no symptoms or signs of inflammatory arthritis.

- Individual at risk of RA on the basis of laboratory abnormalities (eg, ACPA, RF positivity) with no symptoms or signs of inflammatory arthritis.

- Individual at risk of RA on the basis of symptoms of inflammatory arthritis (eg, arthralgia/morning stiffness) but no clinical or imaging evidence of synovitis.

- Individual with synovitis on imaging but no clinically apparent inflammatory arthritis.

- Individual with clinically apparent inflammatory arthritis not yet fulfilling classification criteria for RA.

order to inform clinically relevant developments in outcome prediction and therapy.

The initial remit of the study group was to agree on terminologies to be used during the preclinical and earliest clinically apparent phases of RA and to define important research questions. This report describes this process and its conclusions.

\section{METHODS AND RESULTS}

\section{Terminology for describing phases of RA}

To facilitate discussion about terminologies to be used in the phases of disease before individuals develop RA, a three-stage structured process was followed.

\section{Stage 1}

Brief summaries of widely accepted phases that individuals (may) pass through before the development of RA (box 1) were circulated to participants (19 key researchers in the field; rheumatologists, basic scientists and a patient representative), who were asked to provide descriptive terms for these phases, and to comment on their chosen descriptive terms. Participants were asked to identify whether they felt that there were other phases that should be considered in the context of the development of descriptive terms. Terms that are currently widely used to describe individuals in phases before the development of RA (box 2) were circulated to participants who were asked to state whether they felt the terms were useful and to comment further on them. Fifteen responses were received for stage 1.

\section{Stage 2}

Anonymised data from stage 1 were fed back to participants before a 2-day meeting.

\section{Stage 3}

Eighteen of the participants met during a 2-day workshop. Anonymised responses from previous stages were presented and discussed at the meeting. The following key issues were discussed. First, there was extensive discussion about the terms 'pre-RA' and 'preclinical RA'. It was felt that it was only appropriate to use these in situations in which one knew that the final outcome was RA and when one was looking backwards through the patient's history describing particular phases the patient had gone through. In particular, there was consensus that the term 'pre-RA' suggested that the patient would definitely progress to RA; without a set of risk factors that were $100 \%$ specific it was felt that the term was inappropriate and, as highlighted by the patient representative, of concern to patients and their families if it was used as a shorthand for those individuals identified at high risk of RA.

Second, there was consensus that the terminology used should be able to reflect that the different phases: do not occur in all patients who eventually develop RA (eg, some patients may never have evidence of autoantibodies associated with RA during the preclinical phase of disease); do not necessarily occur in the same order in all patients (eg, some patients may develop autoantibodies before the development of inflammatory joint symptoms and other patients may develop these after the development of such symptoms).

Following these discussions a draft proposal for terminology was developed and this was further refined. The final consensus is shown in box 3 .

\section{Recommendations for reporting of the phases of clinically apparent disease}

Currently there is a diversity of approaches to the dating of 'onset' in cohorts of patients with RA including from: the onset of any joint symptoms related to the current episode including arthralgia/morning stiffness; the onset of self-reported joint swelling; the onset of clinically observed swelling; the time of fulfilment of classification criteria for RA.

During the 2-day meeting, a consensus was reached that, as best practice, in prospective cohort studies the following dates are recorded:

(1) First musculoskeletal symptoms relevant (in the opinion of the assessing rheumatologist) to the current complaint.

(2) First persistent (ie, chronic until presentation) patient reported joint swelling.

(3) Initial fulfilment of criteria for RA (1987 American College of Rheumatology (ACR) $)^{9}$ and 2010 ACR EULAR) ${ }^{10}$ based on data obtained retrospectively from the patient's history.

(4) Initial fulfilment of criteria for RA (1987 ACR and 2010 ACR EULAR) based on the rheumatologist's assessment.

It was recognised that it may not be feasible in all situations to collect all these data. However, it was felt that in all situations investigators should report clearly what they were using as their 'starting point' when reporting symptom/disease durations.

\section{Future research agenda}

During the 2-day meeting a number of areas were identified as being in need of urgent investigation:

Identification of additional risk factors and biomarkers and analysis of interactions between these as individuals progress through different phases of disease

The need to identify additional risk factors and biomarkers for the development of RA was unanimously agreed. Each established and putative factor will have to be clearly defined, in order to harmonise the classification of individuals between cohorts, for example as a precise genotype rather than an allelic odd ratio for a genetic factor. A clear need was identified for an assessment of which phases these were risk factors/ biomarkers for. For example, a particular risk factor may be relevant only to progression to the development of systemic 
Box 2. Information provided to delegates for anonymous comment: commonly used terms that are currently used to describe individuals in phases before the development of RA. Delegates were asked to state whether they felt the terms were useful and to comment further on them.

- Pre-RA

- Preclinical RA

- Inflammatory arthralgia

- Autoantibody-positive arthralgia

- Undifferentiated arthritis

autoimmunity associated with RA, but if assessed in patients in that phase would not be a risk factor for progression though to the development of RA. In contrast, a different risk factor may be relevant to progression from unclassified arthritis to the development of RA. Using the knowledge of risk factors important in each phase, risk stratification and prediction models might identify those individuals who would benefit from intervention applicable to that phase.

\section{Prediction of clinically relevant outcomes in addition to the development of RA}

The heterogeneous nature of RA was acknowledged and it was agreed that, in addition to predicting the development of RA, research should look at biomarkers and risk factors for the rate of progression of joint destruction and the development of extra-articular manifestations of RA (eg, cardiovascular disease and pulmonary fibrosis) that could be identified in individuals 'at risk' of RA. Factors related to remission would also be of interest. The possible influence of the timing and intensity of therapy on these longer-term outcomes should be acknowledged.

\section{Systemic autoimmune phase and site(s) of disease initiation}

The term 'systemic autoimmunity associated with RA' was felt to be the best to describe the phase during which abnormalities in various body compartments can be found preceding the clinical expression of the disease. Although the synovium is the principal site of pathology in the established phase of disease, it may not be the site where the disease is initiated. Systemic immune abnormalities in individuals without joint symptoms, and a lack of immune infiltrates in the synovium during the earliest phase before clinical signs and symptoms of arthritis, ${ }^{7}$ point to other tissues being important in the initiation of adaptive immune reactions. Important tissues for research include bone marrow, lymph nodes, the gut, periodontal tissue, ${ }^{11}$ the lung ${ }^{8}$ and the neuroendocrine system. An initial phase, characterised by systemic autoimmunity without synovial inflammation, may be followed by a shorter phase during which asymptomatic synovitis is present. ${ }^{1213}$ Based on the results of research on various tissues during different stages of the disease, it may be possible to develop specific preventive interventions targeting the breaking of tolerance or the formation of autoantibodies.

\section{Symptoms without clinical arthritis phase}

It was widely recognised that many patients with RA have a period of symptoms likely to be related to the development of inflammatory joint disease before they develop clinical arthritis. Two important areas for research were identified within this
Box 3. Recommendation for terminology to be used

to define specific phases up to the development of RA

- In prospective studies individuals would be described as having:

(a) Genetic risk factors for RA

(b) Environmental risk factors for RA

(c) Systemic autoimmunity associated with RA

(d) Symptoms without clinical arthritis

(e) Unclassified arthritis

(f) RA

- The term 'arthritis' is used to denote clinically apparent soft tissue swelling or fluid (not bony overgrowth alone).

- (a) to (e) can be used in a combinatorial manner for example, an individual may have $(a)+(b)$, or $(a)+(b)+(c)$ or $(a)+(b)+(d)$, etc.

- The prefix 'pre-RA with:' can be used before any/any combination of (a) to (e) but only to describe retrospectively a phase an individual was in once it is known that they have developed RA.

phase. First, the significance of synovial and bone abnormalities as assessed by imaging (eg, ultrasound or MRI), which may detect changes such as synovial thickening, increased synovial vascularity and bone marrow oedema in patients with symptoms without clinical arthritis, as predictors of progression to unclassified arthritis and the development of RA. Second, the importance of identifying symptoms/complexes of symptoms that are associated with the subsequent development of RA.

\section{DISCUSSION}

The characterisation of genetic and environmental risk factors for RA, and the identification of blood-based biomarkers predictive of development of RA in as yet asymptomatic individuals, has led to increasing interest in the development of predictive and preventive strategies that can be used before the onset of symptoms. This comes against a background of considerable research looking at predictive and therapeutic approaches in patients with symptoms before they fulfil the classification criteria for RA. ${ }^{614-19}$ These criteria ${ }^{910}$ have allowed for a common understanding of what we mean by this phase in the patient's journey. There is, however, a plethora of terms currently used to describe individuals in disease phases before the fulfilment of classification criteria for RA, including 'early RA', 'very early RA' and 'pre-RA'. The recommendations in this paper propose guidance on approaches to describe specific phases before the development of RA, which are influenced by our current thinking about aetiopathogenesis. The use of these terms in the manner suggested will facilitate communication between researchers and comparisons between studies, in much the same way that classification criteria facilitate recruitment of patients with similar clinical phenotypes to clinical trials. An important outcome from the meeting was a decision to recommend that the use of the term 'pre-RA' should be limited to very specific situations. To reflect this, the original name of the study group (Study Group for Preclinical and the Earliest Clinically Apparent Phases of Rheumatoid Arthritis) has been changed to Study Group for Risk Factors for Rheumatoid Arthritis 
The terms used to describe the different phases before the development of RA are intentionally broad. Therefore, 'genetic risk factors for RA' and 'environmental risk factors for RA' deliberately do not define any particular genetic and environmental risk factors. 'Systemic autoimmunity associated with RA' does not define any specific immune abnormalities, such as any specific autoantibody, and 'symptoms without clinical arthritis' does not define any specific symptoms. This was a very conscious decision, as restricting these categories by specifying risk factors and features that are currently known will render the nomenclature rapidly redundant in this advancing field. Future research will define additional risk factors and features relevant to each of these phases, beyond current knowledge, and will assess the predictive utility of each of these. In addition, there are currently recognised biomarkers that are known to be present before the development of RA, which are not specifically reflected in the nomenclature. The presence of synovitis on imaging, but not clinically, is an example of this. This is not to suggest that the study group viewed this as an unimportant biomarker. Rather, the nomenclature should provide a framework within which future research can assess at which phases, and for transit to which phases, this, like other biomarkers, is useful.

A number of the research questions that have been defined require new cohorts, of asymptomatic individuals and of patients, to be established and new techniques to be developed to image and collect material from different sites. The identification of uniform core datasets that can be collected in standardised ways across cohorts is an important goal. Furthermore, standard operating procedures for the collection, handling and storage of samples, such as serum and RNA, should be agreed to ensure good and consistent quality of the biomaterial. Given the inherent conflict between the desire to collect material in a uniform and optimal manner and the constraints of working in a clinical environment where such collection is not always feasible, the description of how each sample was collected, handled and stored, will be very important.

As knowledge evolves it is important not to leave its true target audience behind. Individuals without RA need to be informed of the rationale for and value of predicting risk and intervening and the need to have a clear understanding of the risks and benefits of such approaches. Clarity of terminology and the involvement of users through all phases of the research process will help achieve this.

Contributors All authors contributed actively to the development of the recommendation proposed in this manuscript. They all had input in preparing and writing the manuscript. DMG and KR finalised the manuscript. The first two authors contributed equally to the paper.

Funding This work was supported in part by grants from the EULAR, the European BTCure IMl programme, Dutch Arthritis Foundation and ZonMw.

\section{Competing interests None.}

Provenance and peer review Not commissioned; externally peer reviewed.

Author affiliations ${ }^{1}$ Division of Clinical Immunology and Rheumatology, Academic Medical Center, University of Amsterdam, Amsterdam, The Netherlands

${ }^{2}$ Rheumatology Research Group, MRC Centre for Immune Regulation, Institute for Biomedical Research, School of Immunity and Infection, College of Medical and Dental Sciences, University of Birmingham, Birmingham, UK

${ }^{3}$ Department of Rheumatology and Clinical Immunology, University Medical Center Groningen, University of Groningen, Groningen, The Netherlands

${ }^{4}$ Department of Rheumatology and Clinical Immunology, Charite University Hospital, Berlin, Germany

${ }^{5}$ Division of Rheumatology, University of Geneva School of Medicine, Geneva, Switzerland ${ }^{6}$ Rheumatology Unit, Department of Medicine, Karolinska Institute and Karolinska University Hospital, Solna, Sweden

${ }^{7}$ Academic Department of Rheumatology, Division of Immunology, Infection and Inflammatory Diseases, King's College School of Medicine, London, UK

${ }^{8}$ GenHotel, University Evry, Clermont-Ferrand, France
${ }^{9}$ Department of Public Health and Clinical Medicine, Rheumatology, Umeå University, Umeå, Sweden

${ }^{10}$ Section of Musculoskeletal Disease, Leeds Institute of Molecular Medicine, University of Leeds, Leeds, UK

${ }^{11}$ Manchester Academic Health Science Centre,The University of Manchester,

Manchester, UK

12Department of Rheumatology, University Hospital, Zurich, Switzerland

${ }^{13}$ Department of Rheumatology, Erasmus MC, Rotterdam, The Netherlands

${ }^{14}$ Department of Rheumatology, Leiden University Medical Center, Leiden, The Netherlands

${ }^{15}$ Department of Rheumatology, Diakonhjemmet Hospital, Oslo, Norway

${ }^{16}$ Department of Rheumatology, Medical University of Vienna, Vienna, Austria

${ }^{17}$ Department of Immunology, Genetics and Pathology, Uppsala University, Uppsala, Sweden

${ }^{18} \mathrm{Jan}$ van Breemen Research Institute, Department of Rheumatology, Amsterdam, The Netherlands

${ }^{19}$ Department of Internal Medicine 3, University of Erlangen-Nuremberg, Erlangen, Germany

${ }^{20}$ National Rheumatoid Arthritis Society, UK

\section{REFERENCES}

1. van Zeben D, Hazes JM, Zwinderman $A H$, et al. Clinical significance of rheumatoid factors in early rheumatoid arthritis: results of a follow up study. Ann Rheum Dis 1992:51:1029-35.

2. Berglin E, Padyukov L, Sundin U, et al. A combination of autoantibodies to cyclic citrullinated peptide (CCP) and HLA-DRB1 locus antigens is strongly associated with future onset of rheumatoid arthritis. Arthritis Res Ther 2004;6:R303-8.

3. Nielen MM, van Schaardenburg D, Reesink HW, et al. Specific autoantibodies precede the symptoms of rheumatoid arthritis: a study of serial measurements in blood donors. Arthritis Rheum 2004;50:380-6.

4. Rantapää-Dahlqvist S, de Jong BA, Berglin E, et al. Antibodies against cyclic citrullinated peptide and IgA rheumatoid factor predict the development of rheumatoid arthritis. Arthritis Rheum 2003;48:2741-9.

5. Jørgensen KT, Wiik A, Pedersen M, et al. Cytokines, autoantibodies and viral antibodies in premorbid and postdiagnostic sera from patients with rheumatoid arthritis: case-control study nested in a cohort of Norwegian blood donors. Ann Rheum Dis 2008;67:860-6.

6. van de Stadt LA, van der Horst AR, de Koning MH, et al. The extent of the anticitrullinated protein antibody repertoire is associated with arthritis development in patients with seropositive arthralgia. Ann Rheum Dis 2011;70:128-33.

7. van de Sande MG, de Hair MJ, van der Leij C, et al. Different stages of rheumatoid arthritis: features of the synovium in the preclinical phase. Ann Rheum Dis 2011;70:772-7.

8. Klareskog L, Stolt P, Lundberg K, et al. A new model for an etiology of rheumatoid arthritis: smoking may trigger HLA-DR (shared epitope)-restricted immune reactions to autoantigens modified by citrullination. Arthritis Rheum 2006;54:38-46.

9. Arnett FC, Edworthy SM, Bloch DA, et al. The American Rheumatism Association 1987 revised criteria for the classification of rheumatoid arthritis. Arthritis Rheum 1988;31:315-24.

10. Aletaha D, Neogi T, Silman AJ, et al. 2010 Rheumatoid arthritis classification criteria: an American College of Rheumatology/European League Against Rheumatism collaborative initiative. Ann Rheum Dis 2010;69:1580-8.

11. de Pablo P, Chapple IL, Buckley CD, et al. Periodontitis in systemic rheumatic diseases. Nat Rev Rheumatol 2009;5:218-24.

12. Kraan MC, Versendaal $H$, Jonker $M$, et al. Asymptomatic synovitis precedes clinically manifest arthritis. Arthritis Rheum 1998;41:1481-8.

13. Gent YY, Voskuyl AE, Kloet RW, et al. Macrophage PET imaging as biomarker for preclinical rheumatoid arthritis. Arthritis Rheum 2012;64:62-6.

14. Filer $\mathbf{A}$, de Pablo P, Allen $\mathrm{G}$, et al. Utility of ultrasound joint counts in the prediction of rheumatoid arthritis in patients with very early synovitis. Ann Rheum Dis 2011;70:500-7.

15. van der Helm-van Mil AH, Detert J, le Cessie S, et al. Validation of a prediction rule for disease outcome in patients with recent-onset undifferentiated arthritis: moving toward individualized treatment decision-making. Arthritis Rheum 2008; 58:2241-7.

16. Verstappen SM, McCoy MJ, Roberts C, et al. Beneficial effects of a 3-week course of intramuscular glucocorticoid injections in patients with very early inflammatory polyarthritis: results of the STIVEA trial. Ann Rheum Dis 2010;69:503-9.

17. Machold KP, Landewé R, Smolen JS, et al. The Stop Arthritis Very Early (SAVE) trial, an international multicentre, randomised, double-blind, placebo-controlled trial on glucocorticoids in very early arthritis. Ann Rheum Dis 2010;69:495-502.

18. van Dongen $\mathbf{H}$, van Aken J, Lard LR, et al. Efficacy of methotrexate treatment in patients with probable rheumatoid arthritis: a double-blind, randomized, placebocontrolled trial. Arthritis Rheum 2007;56:1424-32.

19. Bos WH, Dijkmans BA, Boers M, et al. Effect of dexamethasone on autoantibody levels and arthritis development in patients with arthralgia: a randomised trial. Ann Rheum Dis 2010;69:571-4. 